\title{
Les collections équestres du Centre des monuments nationaux : les châteaux de Bouges et de Maisons
}

Morwena Joly-Parvex, Clotilde Roy, Grégoire Cuny et Céline do Paço

\section{OpenEdition}

Journals

Édition électronique

URL : http://journals.openedition.org/insitu/11957

DOI : 10.4000/insitu. 11957

ISSN : 1630-7305

Éditeur

Ministère de la culture

Référence électronique

Morwena Joly-Parvex, Clotilde Roy, Grégoire Cuny et Céline do Paço, « Les collections équestres du Centre des monuments nationaux : les châteaux de Bouges et de Maisons », In Situ [En ligne],

27 | 2015, mis en ligne le 02 novembre 2015, consulté le 30 avril 2019. URL : http:// journals.openedition.org/insitu/11957 ; DOI : 10.4000/insitu.11957

Ce document a été généré automatiquement le 30 avril 2019.

\section{(c) $(1) \Theta \theta$}

In Situ Revues des patrimoines est mis à disposition selon les termes de la licence Creative Commons Attribution - Pas d'Utilisation Commerciale - Pas de Modification 4.0 International. 


\title{
Les collections équestres du Centre des monuments nationaux : les châteaux de Bouges et de Maisons
}

\author{
Morwena Joly-Parvex, Clotilde Roy, Grégoire Cuny et Céline do Paço
}

1 Parmi les monuments dont la gestion est confiée au Centre des monuments nationaux (CMN), deux monuments sont plus particulièrement marqués par la présence du cheval: le château de Bouges (Indre) et celui de Maisons-Laffitte (Yvelines).

2 Au château de Bouges, la passion éclairée du dernier maître des lieux, Henri Viguier - qui y aménagea au début du $x^{e}$ siècle une luxueuse écurie - a permis de constituer une des plus splendides collections françaises d'équipements, de voitures et de harnachements. À Maisons, les collections hippiques rassemblées ne sont pas liées aux propriétaires historiques du château, mais ont été constituées par l'Association du musée du Cheval de courses, en liaison avec l'histoire des écuries du château mais surtout avec celle de l'hippodrome de Maisons-Laffitte.

3 Ces deux exemples permettent d'évoquer les deux grands types de collections équestres, celles mémorielles, attachées à une personnalité, une pratique ou un lieu, et celles muséales, rassemblées à la faveur des opportunités et des goûts des collectionneurs hippiques. Cette confrontation permet à la fois de dresser un bilan de l'évolution des présentations des collections au sein des monuments et d'esquisser un débat théorique plus large, au-delà des seules collections équestres du Centre des monuments nationaux.

\section{Le château de Bouges : historique de la constitution des collections}

4 Le château de Bouges est situé dans le département de l'Indre, à une vingtaine de kilomètres de Châteauroux, non loin du château de Valençay. Il a été bâti entre 1765 et 1773 sur les ruines d'une maison forte, par Claude Charles François Leblanc de Marnaval 
et a été légué en 1967 par Henri Viguier à la Caisse nationale des monuments historique et des sites ${ }^{1}$.

Par son plan rectangulaire et son organisation en pavillon, le château de Bouges, dès l'origine, a été conçu comme un pavillon de chasse et une demeure de plaisance ${ }^{2}$. L'espace des communs qui abrite l'écurie est situé en contrebas du côté sud du château. Le bâtiment forme un L autour d'une cour fermée dans sa partie nord par le mur de soutènement du château. L'ensemble est composé d'un corps principal et d'une orangerie en retour. Les restaurations et le réaménagement du corps principal sont essentiellement dus à Henri Viguier. Il entreprend les travaux dès 1918 pour y abriter une écurie, une sellerie d'honneur et une sellerie de travail, puis investit l'orangerie pour y remiser sa collection de voitures hippomobiles. Les installations de l'écurie sont d'un grand raffinement. Le répertoire décoratif qui s'y déploie est simple et l'usage exclusif du bois de chêne en assure l'unités.

Né en 1877, Henri Viguier est président-directeur général des magasins du Bazar de l'Hôtel de Ville jusqu'au début des années 1960. Il épouse Marie Claire Renée Normand en 1906. Le couple achète Bouges en 1917 et entreprend de restaurer et de remeubler le château, dépecé par ses propriétaires précédents. Mais la grande passion d'Henri Viguier demeure avant tout le cheval. Membre de la Société des guides et de la Société des courses de Châteauroux, il possède sa propre écurie de course, portant casaque bleue et jonquille. Ses loisirs favoris sont la chasse et l'attelage (fig. 1), qu'il pratique jusqu'en 1967, mettant en œuvre les techniques les plus raffinées et entretenant un patrimoine de grande valeur créé par les meilleurs artisans.

Figure 1

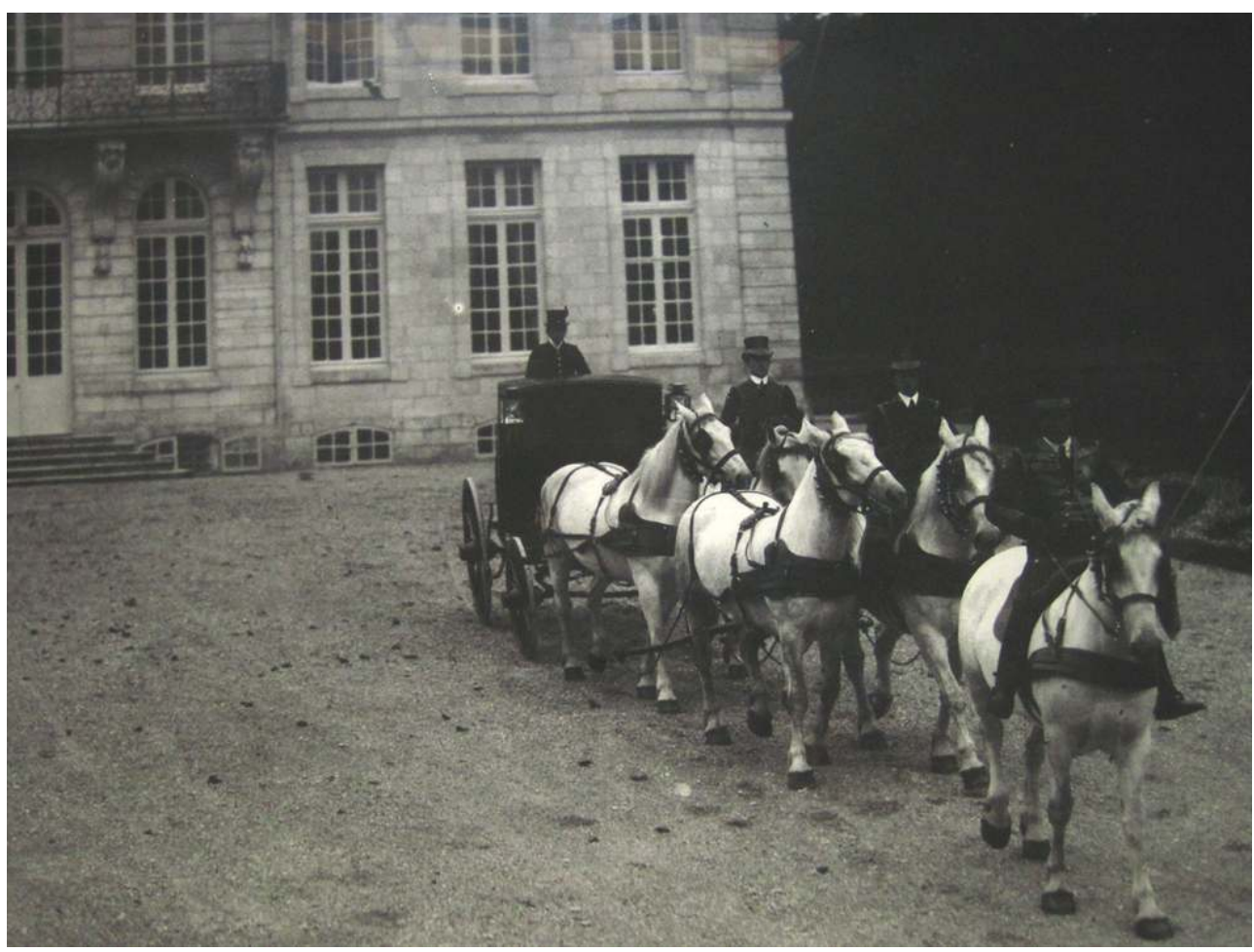

Coupé de d'Aumont, devant l'entrée principale du château de Bouges.

Collection particulière. (c) Centre des monuments nationaux, 2004. 


\section{Les collections dédiées au cheval}

La pièce maîtresse des écuries, la plus luxueuse aussi, est sans conteste la sellerie d'honneur. C'est là que sont entreposés les harnachements les plus précieux qui participent au prestige du propriétaire des lieux. Il s'agit d'une pièce de plan carré, lambrissée et décorée de gravures de scènes de chasse et de trophées. Tout concourt à donner à cet espace une atmosphère résolument sportive et cynégétique (fig. 2). Au centre, un meuble-vitrine d'origine contient mors et étriers qui bénéficient d'une présentation quasi muséographique que l'on retrouve dans les selleries contemporaines du château d'Espeyran (Gard) ou de Valmirande (Haute-Garonne). On y trouve des mors d'attelage à ballon et canons droits, des mors de brides de type "Lhotte» ou "Wellington", des mors de filet "Chantilly», etc. ${ }^{4}$. Cette remarquable variété typologique est équivalente à celle des harnais et des selles: selles de postillon, selles anglaises, selles d'amazone achetées en Angleterre ou auprès de la maison Hermès. Pour les harnais, deux grands types se distinguent : les harnais de ville avec leur collier et bride à frontal bleu et jonquille pour les attelages à deux ou quatre chevaux et les harnais sportifs, à bricoles et grelottières pour les attelages «en poste». Tous sont au chiffre de leur propriétaire. L'ensemble est complété par des bottes de cavalier ou de postillon ou des pièces plus atypiques comme un petit traîneau anversois.

Figure 2

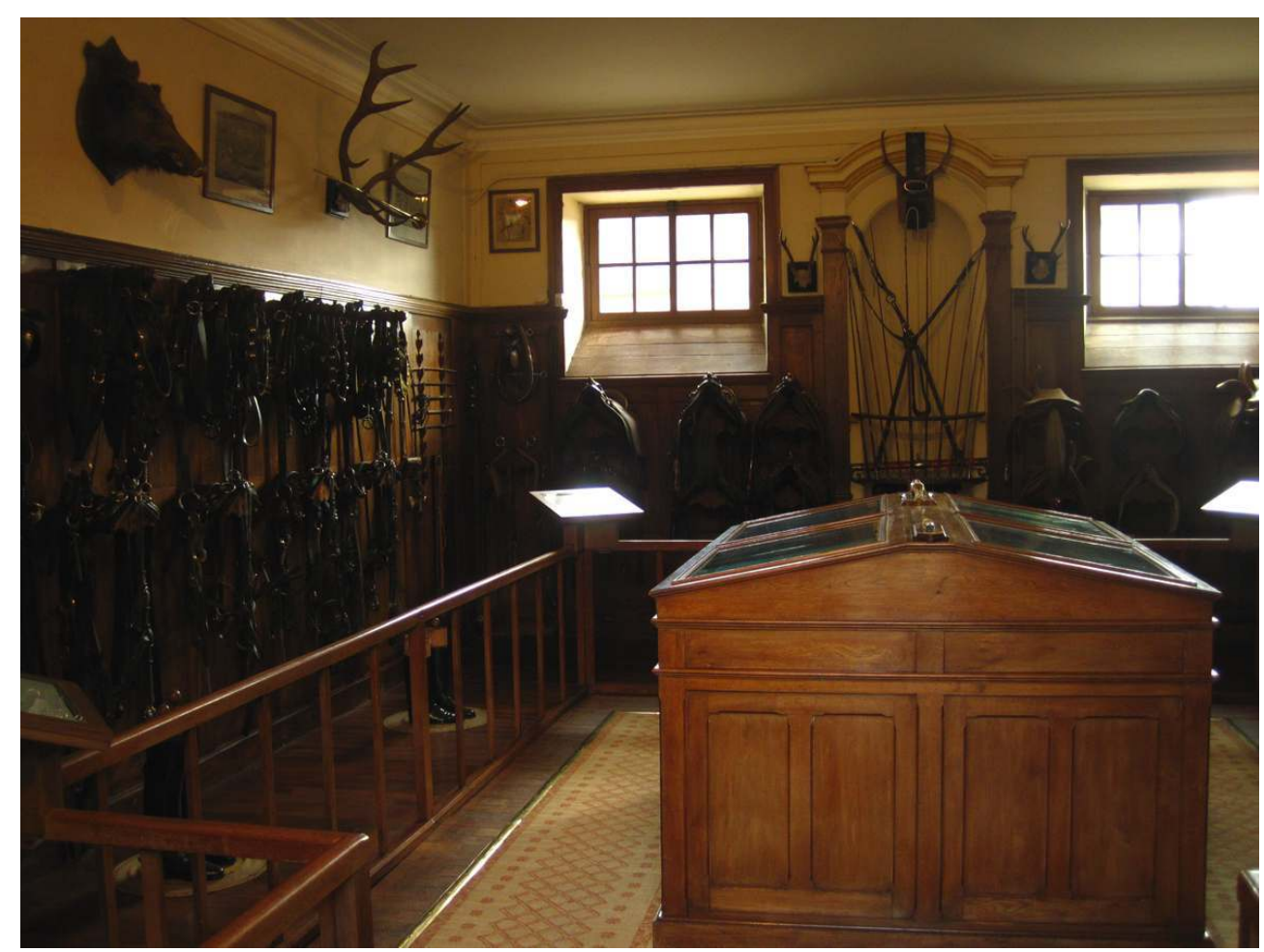

La sellerie d'honneur du château de Bouges, vue générale.

Phot. Cuny, G. (c) Centre des monuments nationaux, 2012.

8 On accède à la seconde sellerie, dite de travail, par l'autre côté du bâtiment. Cette disposition prévoit donc une façade de prestige, face au château, par laquelle on accède à 
l'écurie et à la sellerie d'honneur, alors que les soins et la préparation des chevaux se déroulent dans la basse-cour. On y retrouve beaucoup d'harnachements, souvent d'un type plus commun que dans la sellerie d'honneur, mais aussi plusieurs équipements utilitaires et instruments de soins du début du siècle comme une rare tondeuse mécanique, un four à mash ${ }^{5}$, une presse à linge ou un nécessaire de soins complet comprenant tricoises, instruments de pansage, arrache-crins, étrille, râpe, etc.

Les quatorze voitures hippomobiles ont été transférées dans la grange aménagée en remise, à l'arrière de l'écurie. Elles sont toutes peintes aux couleurs des Viguier, caisse bleue et train jonquille. Les voitures de maître livrées par de grandes maisons parisiennes côtoient des voitures de service construites par des carrossiers provinciaux et destinées soit aux activités domestiques, soit à la chasse ${ }^{6}$. Des voitures emblématiques se distinguent, telle le road coach, version privée du coach, qui est destiné aux longs trajets routiers. L'intérieur est garni de cuir capitonné et de tissu vert et des miroirs sont fixés au revers des volets des portières. Il est utilisé par une élite passionnée et fortunée lors de parties de campagne, de pique-niques élégants, ou de courses hippiques où il sert de tribune privée. Il s'attelle toujours à quatre chevaux, menés par le propriétaire dont il atteste la fortune et les qualités d'homme de cheval accompli. La fonction du break grand modèle est en revanche plus clairement cynégétique (fig. 3), même si celui de Bouges servait fréquemment aux usages domestiques. Il est signé Bail jeune frères. Lors des chasses on attelait également une voiture à gibier, comme celle fabriquée par Duchatelle à Creil, équipée de plusieurs centaines de crochets pour y suspendre les prises, ainsi parfois qu'une petite voiture légère et robuste comme la meadowbrook car ${ }^{7}$, exemplaire signé Dousserin à Vierzon.

Figure 3

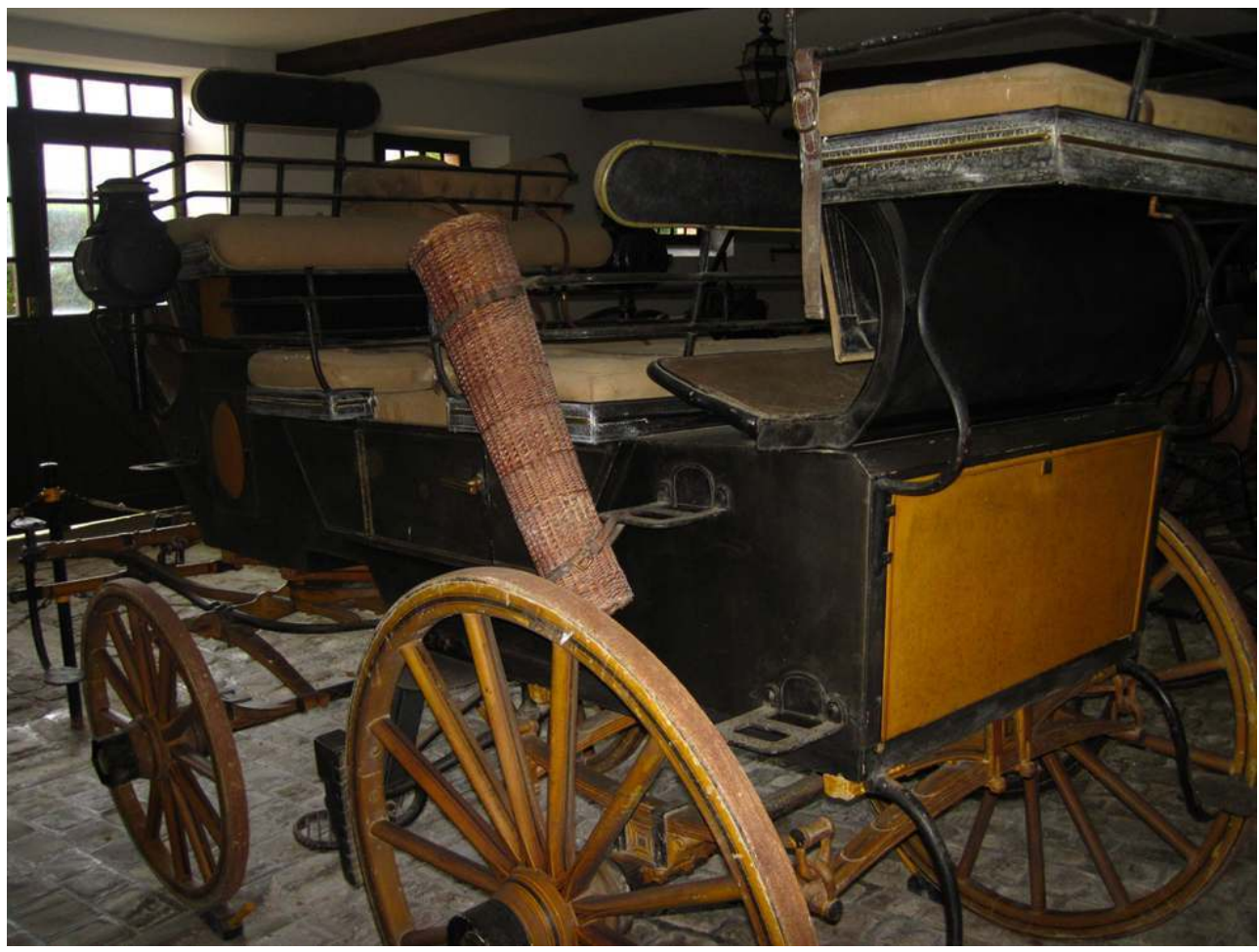

Break grand modèle, par Bail jeune frères à Paris, vers 1900.

Phot. Cuny, G. (c) Centre des monuments nationaux, 2012. 


\section{La valorisation des collections et ses enjeux}

10 situation parfois approximatives, les pratiques actuelles favorisent désormais les présentations claires et conformes aux usages des pièces présentées. Elles doivent aussi ne pas perturber la lisibilité des espaces, ni la grande cohérence des collections et de leur disposition d'origine. Ainsi surgit d'emblée la difficulté d'apporter au public des informations précises et pertinentes liées aux fonctions et à la typologie complexe des patrimoines du cheval sans porter préjudice à l'harmonie générale.

11 Il y a quelque temps encore, les supports de médiation étaient constitués de grands panneaux disposés au milieu des espaces historiques: ce dispositif était utile mais disgracieux. Or, ces dernières années, le CMN a acquis un savoir-faire reconnu dans le domaine des nouvelles technologies et des modes de valorisation innovants. Le département des publics a développé, pour de nombreux monuments, des outils numériques d'aide à la visite et des dispositifs de réalité augmentée. Pour les écuries de Bouges, ces outils auraient l'immense avantage d'apporter une information presque illimitée aux visiteurs, notamment sous forme multimédia et interactive sur des supports numériques portatifs, sans que l'authenticité des espaces et de la présentation des collections en soit nullement affectée. Ainsi, les différentes formes d'attelage pourraient y être expliquées sous forme de schémas dynamiques et illustrées par des photographies d'archives. Le multimédia aurait, en outre, l'avantage de pouvoir réintroduire par l'image et le son une trace des hommes, des chevaux et de leurs pratiques communes dans des lieux aujourd'hui sans vie.

12 Les écuries de Bouges et leurs collections sont l'œuvre d'un amateur éclairé et d'un collectionneur passionné. Henri Viguier rassembla ce qui se faisait alors de mieux en matière d'équipement, d'harnachement et de voiture, qu'il mit au service des pratiques équestres les plus élaborées. Le cheval était intégré à toutes les activités du domaine : celles du service et des tâches quotidiennes comme celles des loisirs les plus élitistes, attelage sportif, vénerie, etc. (fig. 4). Il en résulte une collection équestre d'une grande unité, qui forme avec le domaine et le château un ensemble très cohérent. C'est cette unité, associée à la recherche de l'excellence et mise au service d'un art de vivre tombé en désuétude, qui fait des collections de Bouges un ensemble de grand intérêt patrimonial. Or, l'État et plus particulièrement le CMN, qui en a aujourd'hui la charge, conformément à la volonté d'Henri Viguier, est face à de multiple défis : conserver des collections très fragiles dans des conditions qui ne peuvent pas toujours, par nature, répondre aux normes muséales, présenter et valoriser des collections dans leur cadre d'origine sans nuire à l'harmonie de l'ensemble, tout en tenant compte d'un contexte budgétaire très contraint. Mais ces défis, sans nul doute, sauront être surmontés pour que se transmettent longtemps encore l'héritage et la passion d'Henri Viguier. 


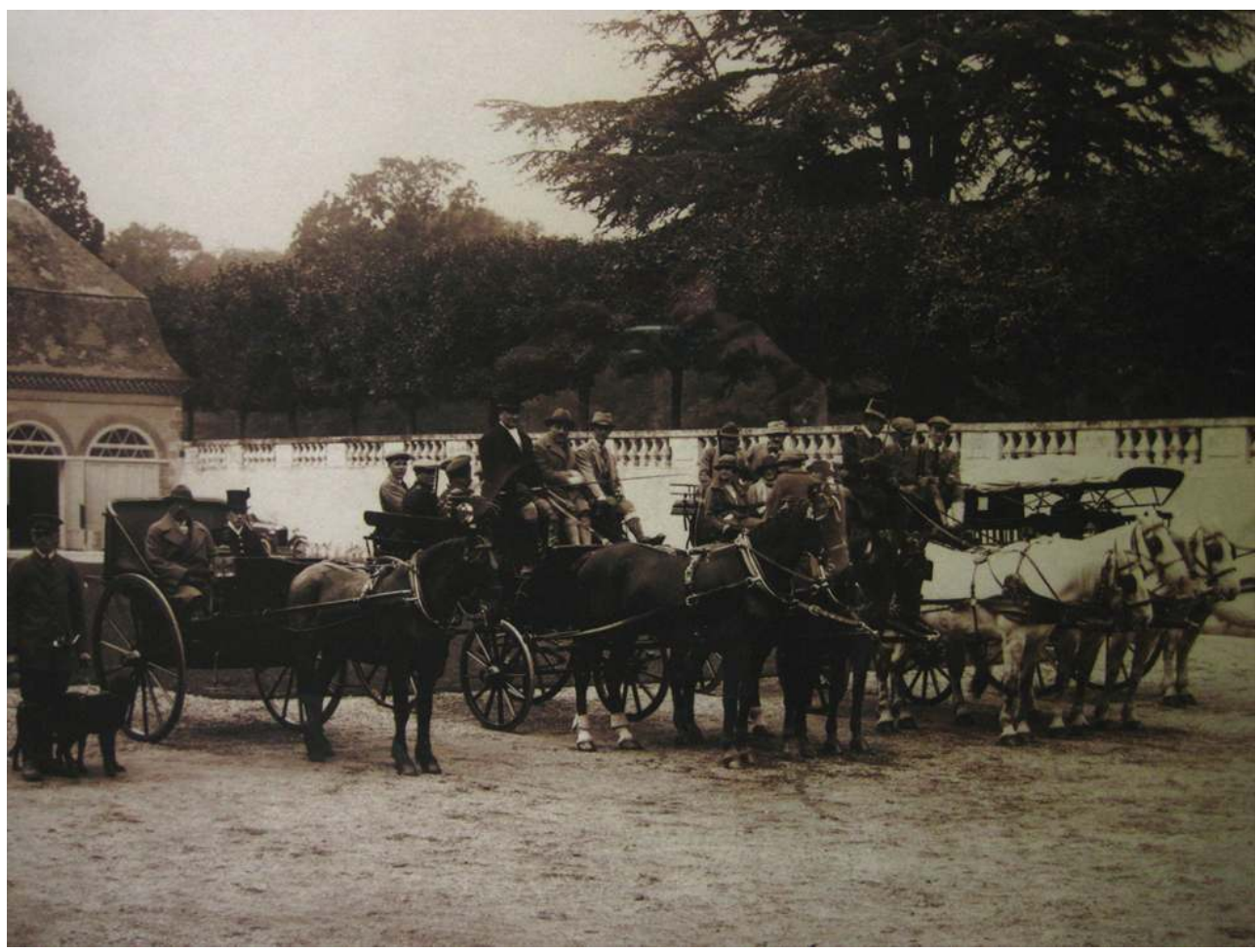

Départ à la chasse devant les écuries de Bouges.

Collection particulière. (C) Centre des monuments nationaux, 2004

\section{Le château de Maisons : historique de la constitution des collections}

À l'occasion de la donation au château de Maisons de l'ensemble de la collection constituée par l'Association pour le musée du Cheval de courses (2010), une campagne d'inventaire a été menée par la direction scientifique du Centre des monuments nationaux. Cette collection hippique d'environ 200 biens culturels est exposée dans les sous-sols du château aux côtés des collections anciennement entrées et de dépôts consentis par France Galop.

Les propriétaires du château de Maisons occupent un rôle fondamental dans l'histoire de la « cité du cheval ». Situé non loin de la résidence de Saint-Germain-en-Laye, où séjourne régulièrement Louis XIII, le château construit par François Mansart (1598-1666) permet à René de Longueil (1593-1677) d'accueillir de façon prestigieuse le roi et sa cour lors de chasses organisées plusieurs fois par semaine. Le château, doté de dépendances aujourd'hui disparues, possède de remarquables écuries, considérées comme "les plus belles du royaume » selon l'ambassadeur de Suède. Ces écuries monumentales, situées dans l'avant-cour, peuvent accueillir un nombre important de chevaux ${ }^{8}$. Magnifié par une coupole, le manège sert au dressage et à l'entraînement. Lorsque le comte d'Artois achète en 1777 le château de Maisons au marquis de Soyecourt, dernier descendant de la famille Longueil, c'est à la fois en raison de la noblesse du château mais aussi pour ses écuries suffisamment importantes pour loger ses chevaux et son écurie de course. Anglomane, le 
comte d'Artois y constitue une écurie anglaise de 20 à 30 chevaux et fait établir des pistes d'entraînement sur les prairies qui jouxtent le château le long de la Seine'.

Jacques Laffitte, propriétaire du château de Maisons en 1818, joue un rôle clé dans le développement de l'activité hippique à Maisons ${ }^{10}$. Devant faire face à des difficultés financières considérables, Laffitte lance en 1834 une grande opération immobilière paysagère en lotissant le parc du château, donnant naissance à la colonie de Maisons. Les écuries sont alors détruites pour fournir à bas prix des matériaux aux acquéreurs des lots découpés dans le parc. Seul est aujourd'hui conservé un magnifique abreuvoir traité en rocaille, dans les sous-sols de l'une des villas de l'avenue Carnot. Le site de Maisons offre un cadre propice au développement des courses non loin de Paris. Laffitte espère qu'en y implantant les courses, il renforcera l'attrait de la ville. Pour cela il s'appuie sur deux de ses proches, passionnés de sports hippiques: son neveu Charles Laffitte et son gendre Napoléon Joseph Ney, prince de la Moskova, qui a épousé sa fille Albine. Il lui faut construire un hippodrome et des équipements nécessaires à l'entraînement des chevaux. La première réunion officielle de courses hippiques a lieu le 28 juillet 1828 le long de la Seine. La deuxième a lieu lors de l'inauguration de la « colonie de Maisons-sur-Seine » en 1834. L'histoire de l'implantation des courses à Maisons est complexe et ponctuée de rebondissements ${ }^{11}$, mais il est certain que le château de Maisons et ses écuries prestigieuses, puis le lotissement de son parc, ont largement contribué au développement des activités hippiques.

Dès son acquisition par l'État en 1905, le château, sauvé de la démolition par une mobilisation très forte des Mansonniens, se voit chargé de transmettre la mémoire de cette histoire équestre. Organisée par le syndicat d'initiative de Maisons-Laffitte avec le concours de la Société des amis du château de Maisons, la première exposition dédiée aux courses en France est inaugurée en 1926. La seconde, organisée en 1928, est consacrée à Alfred de Dreux (1810-1860), première exposition monographique du célèbre peintre de chevaux. À la suite de ces manifestations, des œuvres importantes entrent dans les collections du château dont le tableau Atalanta, donné en 1932 (fig. 5). 
Figure 5

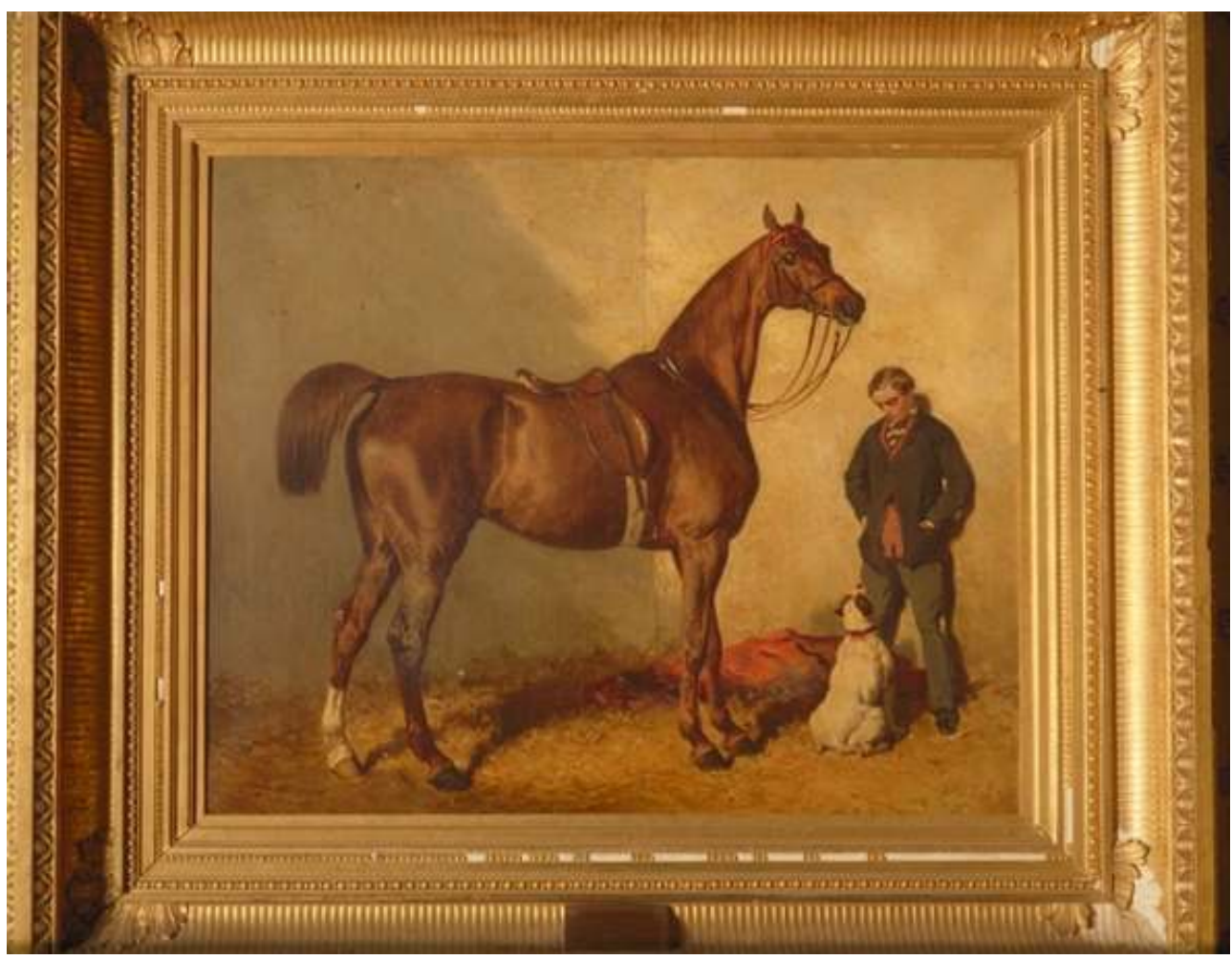

Alfred de Dreux, Atalanta.

Phot. Roy, C. (C) Centre des monuments nationaux, 2004.

17 Si l'on réfléchit dès l'après-guerre à un projet de musée des courses afin de répondre au désir exprimé par la Société sportive d'encouragement et par la municipalité de MaisonsLaffitte, la création d'une association permettant de constituer des collections n'intervient qu'en 1988, sous le nom d'Association pour le musée du Cheval de courses. Il s'agit avant tout de mettre en valeur l'importance historique et patrimoniale de la cité équestre et le musée dédié aux courses s'inscrit dans un contexte de perte de vitesse des activités hippiques à Maisons. Après Saratoga Springs (National Museum of Racing, 1950) aux États-Unis et Newmarket (National Horseracing Museum, 1983) en Angleterre, Maisons-Laffitte inaugure ce musée le $1^{\mathrm{er}}$ juin 1990. De lourds travaux d'aménagement du niveau des douves sont nécessaires. Ils conduisent à la destruction de la chaufferie du XIx ${ }^{e}$ siècle. 
Figure 6

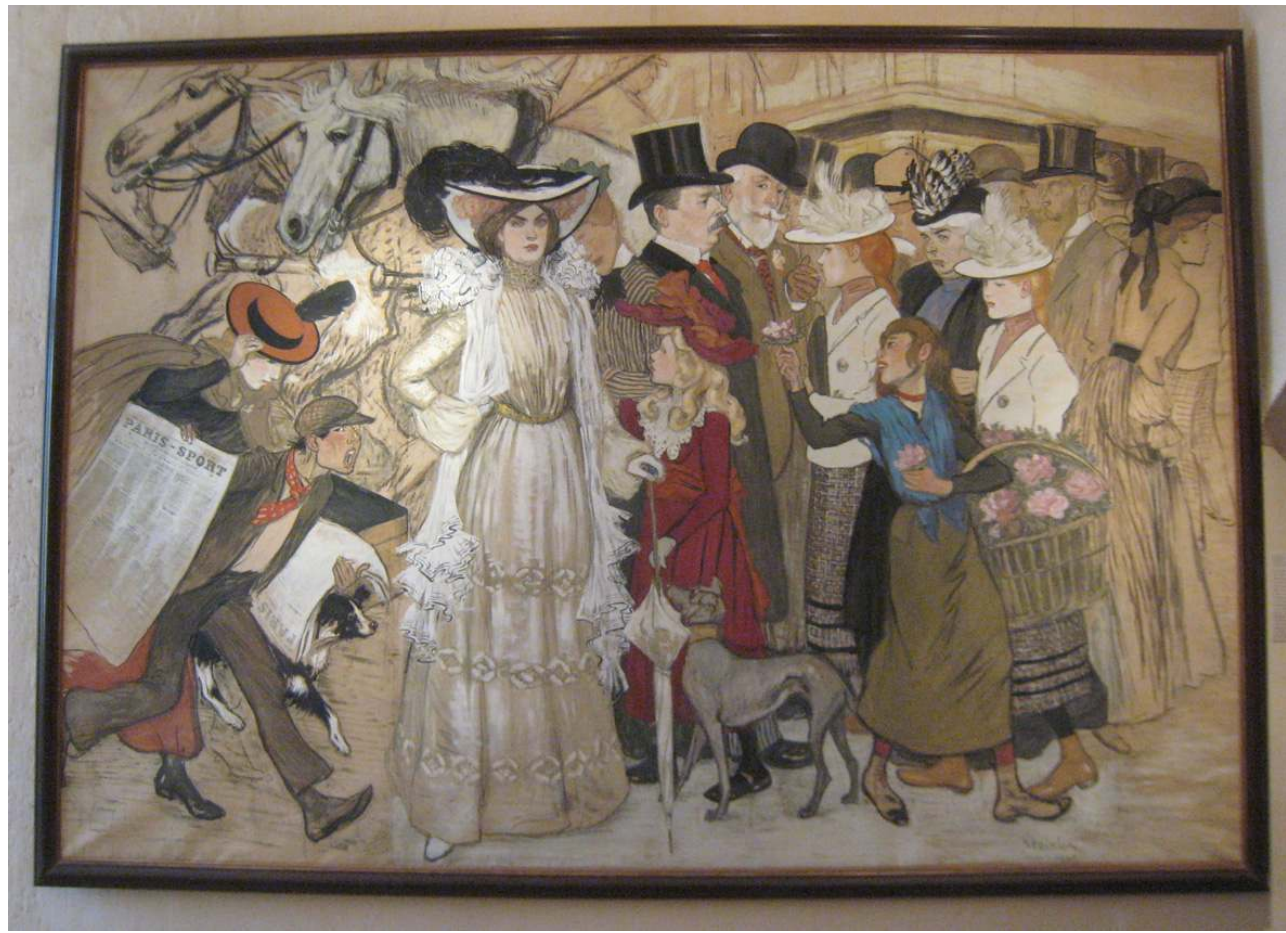

Steinlen, Le Boulevard, 1902.

Phot. Roy, C. (c) Centre des monuments nationaux, 2004.

Le musée associatif, présidé par le baron de Montesquieu, est conçu par Guy Thibault. Dans son organisation, son fonctionnement, ses collections, il est totalement indépendant du château ${ }^{12}$. Il renferme alors quatre œuvres majeures : l'Atalanta d'Alfred de Dreux, Le Boulevard, monumentale gouache de Steinlen (fig. 6), datée de 1902, représentant le public des hippodromes parisiens, Les courses d'Ascot, de Jacques-Émile Blanche (fig. 7) qui nous introduit dans le monde aristocratique et coloré des fameux meetings royaux chers aux Britanniques organisés dans cette ville et Impatience d'avenir, un groupe de jeunes chevaux au galop sculpté par Idel Ianchelevici, qui ne fait plus actuellement partie des collections. 


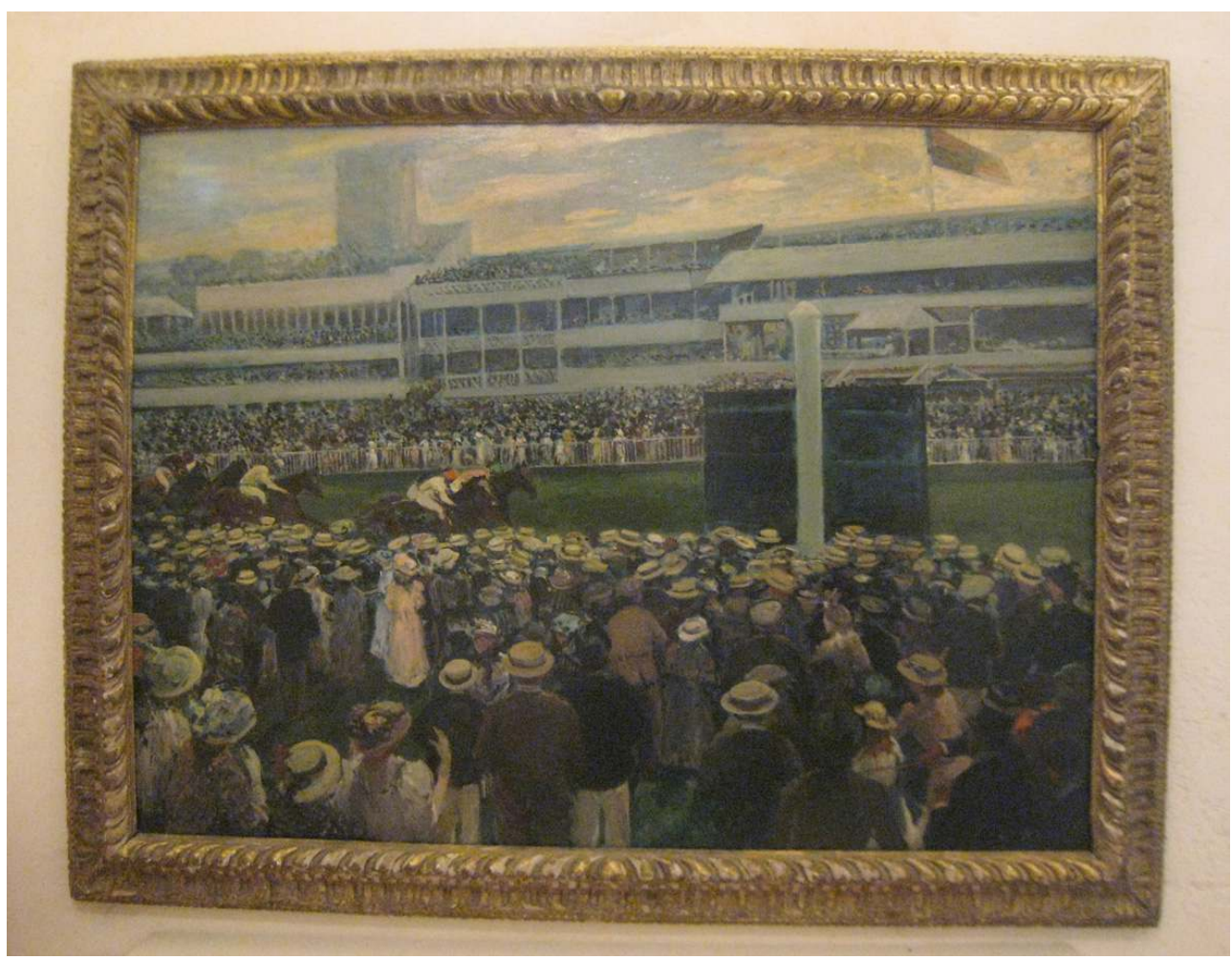

Jacques-Émile Blanche, Les courses à Ascot.

Phot. Roy, C. (c) Centre des monuments nationaux, 2004.

L'idée originelle du musée est de raconter la vie et la carrière du cheval de course, de rassembler et d'exposer des documents, des œuvres d'art, des objets et des souvenirs d'intérêt historique appartenant au patrimoine des courses et des activités annexes, de retracer l'histoire des courses en France des origines à nos jours, de présenter l'organisation de l'élevage et des courses de chevaux en France, d'expliquer le fonctionnement des paris et leur histoire. Les objectifs de l'époque, tels qu'ils sont exprimés dans les communiqués de presse, sont vastes puisqu'il s'agit de favoriser la redécouverte du cheval par l'homme, de susciter de nouvelles vocations (de propriétaires, entraineurs, jockeys, amoureux du cheval et de son environnement sportif) et de faire mieux connaître les courses ainsi que de rappeler le rôle de Maisons-Laffitte dans l'histoire des courses en France ${ }^{13}$. Parmi les membres fondateurs se trouvent ainsi la municipalité de Maisons-Laffitte, la Société sportive d'Encouragement, la Société des steeple-chases de France, la Société de sport de France, la Fédération nationale des Sociétés de courses de France, la Société d'encouragement, le Pari mutuel urbain (PMU), le Pari mutuel hippodrome (PMH) et l'agence de communication INTERACT ${ }^{14}$.

20 Acquises sur le marché de l'art, prêtées ou données par des collectionneurs privés, les collections sont donc à la fois des témoins de pratiques culturelles et sociales, les souvenirs côtoient les œuvres d'art. La collection rassemble peintures, sculptures, œuvres graphiques, photographies, éléments de harnachement, équipement du jockey, une table d'opération pour chevaux, du matériel de maréchal-ferrant, une balance pour le pesage du jockey, des tourniquets compteurs, des trophées, des jouets ainsi que des documents d'archives privées et des ouvrages (contrats, stud-book, règlements de courses, le 
périodique Chronique du Turf de 1906 à 1968), dont il n'est pas toujours aisé de retrouver l'historique ou à défaut un minimum d'informations documentaires. La collection couvre les différents aspects du monde des courses : de l'élevage au soin, de l'entraînement à la course et à l'architecture hippique. Composé autour de sections évoquant aussi bien l'élevage dans sa dimension socio-économique que le monde hippique et sa sociabilité, le noyau essentiel de la collection reste toutefois dédié à la représentation du cheval de course. Cette présentation est ainsi particulièrement représentative d'une manière de collectionner et de concevoir l'hippisme à la fin du $\mathrm{xx}^{\mathrm{e}}$ siècle.

Certains ensembles homogènes méritent une attention particulière, telle la collection de portraits de chevaux comme Atalanta par de Dreux, Salvator (prix du Jockey Club 1875) de Sturgess, Moia (Prix de Diane 1913) de le Nail, Sao Paulo (Grand prix de Deauville) de Frank Elim, Coligny (Grand Prix du printemps 1929) de Georges Busson. La section consacrée à la représentation du galop suscite elle aussi l'intérêt. La question de la représentation du galop volant, bien qu'incomplète, est évoquée par une série d'estampes du XIX siècle non exposées ${ }^{15}$ mais conservées au château, ainsi que par des œuvres plus tardives de Georges Malissard (fig. 8). Quant au Boulevard de Steinlen, esquisse préparatoire au décor réalisé en céramique de Sarreguemines pour l'hippodrome de Saint-Ouen (actuellement conservé à l'hippodrome d'Enghien-les-Bains), son intérêt est indéniable, puisqu'elle permet d'évoquer le public d'un hippodrome parisien à travers les figures du vendeur de journaux (Paris-Sport), de la bouquetière, d'élégants messieurs.

Figure 8

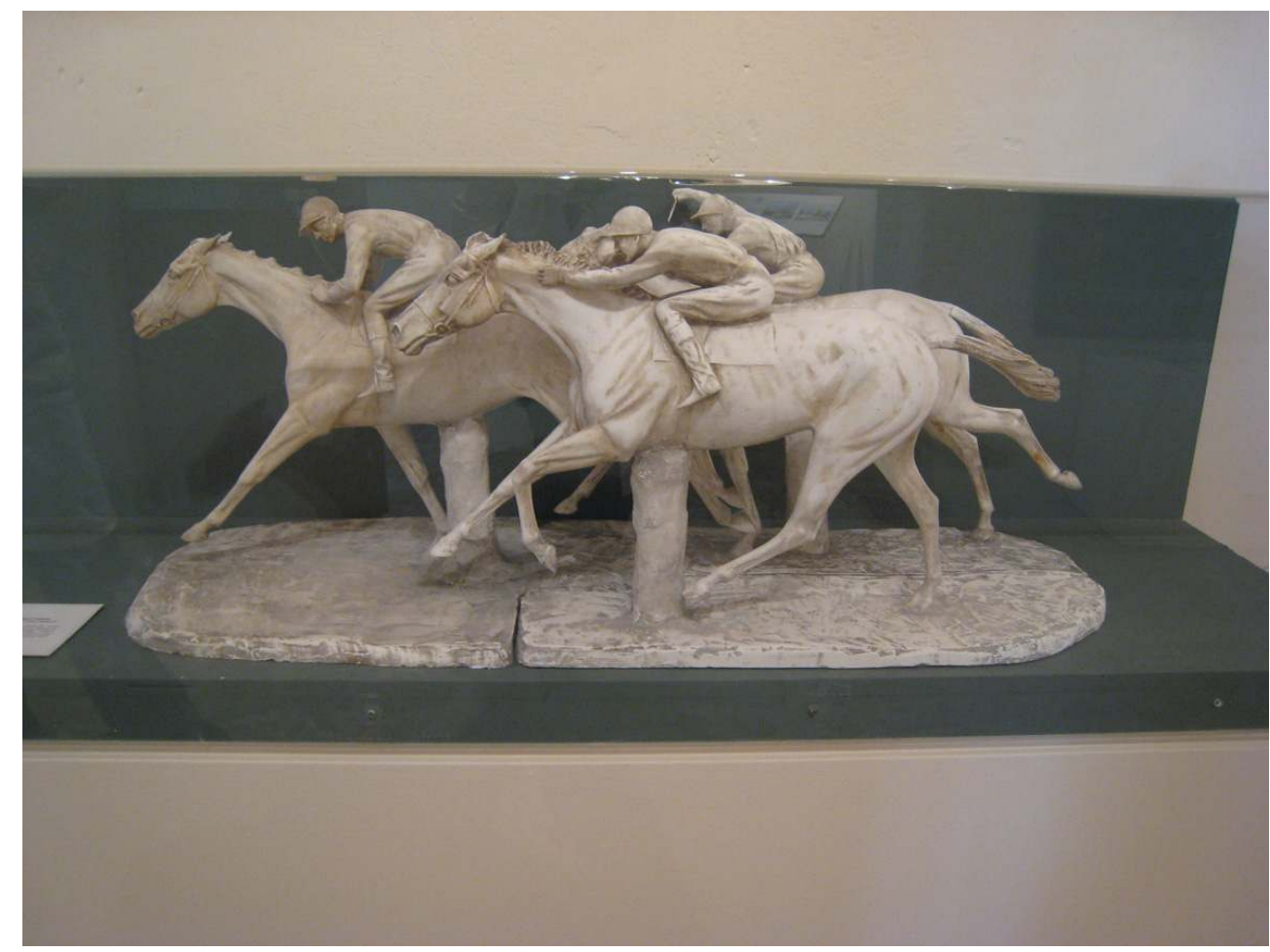

Georges Malissard, Trois chevaux luttant à l'arrivée.

Phot. Roy, C. @ Centre des monuments nationaux, 2010.

Si ces œuvres et témoignages rassemblés méritent incontestablement d'être présentés au public, il n'est pas sûr que ce musée du Cheval de course soit véritablement à sa place 
dans les sous-sols du château de Maisons. La muséographie du lieu, aujourd'hui obsolète, invite à poser plus largement la question de la pertinence d'une exposition permanente de collections équestres rassemblées par des amateurs de courses.

\section{La valorisation des collections équestres dans les monuments : enjeux et perspectives}

Que ce soit à Maisons ou à Bouges, l'évolution des présentations des collections équestres au fil du temps n'est pas sans poser quelques questions. Celles-ci sont d'abord d'ordre muséographique au sens large, puisque le terme de "présentation » lui est généralement préféré au sein des monuments. Deux éléments se dégagent assez nettement. D'une part la présentation de type mémoriel, comme à Bouges, tend à se figer dans une « muséographie » qui semble attirer irrésistiblement un dispositif de type muséal. Ainsi, mise à distance et panneaux-cartels apparaissent dans la sellerie d'honneur (voir fig. 2), ce qui n'est pas sans brouiller la compréhension du lieu, puisqu'il devient davantage un lieu d'exposition qu'un lieu de vie lié à la pratique du cheval. Le phénomène se reproduit dans les écuries, vidées de leur occupant chevalin, où la présentation d'éléments sous vitrine a semble-t-il pour but de combler ce vide. D'autre part, la présentation de type muséal au sein d'un monument, comme à Maisons, nuit considérablement à la lecture des espaces domestiques du château. Le musée du Cheval, coincé entre la cuisine et la salle de bains, espaces qui tendent à être plus ou moins remeublés au fil du temps, se développe aujourd'hui sans lien cohérent avec le monument. L'insertion d'une présentation de type muséal fonctionne mieux dans le cas du musée du Cheval de Spa (Belgique) - haut lieu du sport équestre et des concours hippiques en Europe - car un espace d'exposition des collections a été aménagé dans les anciennes écuries de la reine Marie-Henriette, en cohérence avec les pièces dont la fonction est très identifiée. Ainsi, l'atelier du maréchalferrant accueille très naturellement les machines-outils de l'artisan. Les éléments de sellerie sont mis en scène selon leur valeur d'usage, alors que les collections rassemblées par les amateurs de courses - dont l'hétérogénéité est identique aux collections de Maisons - sont exposées dans des salles spécialement conçues à cet effet. Il n'existe donc pas de hiatus entre le monument et la présentation des collections car chaque espace a été traité différemment.

L'autre question concerne les collections constituées dans le cadre d'une présentation de type muséal. Les collections équestres sont par nature hétérogènes : œuvres d'art, biens culturels attestant de l'évolution sociologique du monde hippique, archives, estampes, photographies... La cohabitation des œuvres et des objets n'est pas aisée, ni visuellement, ni sur le plan du discours. Si l'on parcourt cavalièrement les grands musées consacrés au cheval, les choix effectués révèlent une claire tension au sein des présentations. Dans l'un des modèles du genre, celui de Saratoga, dans l'État de New York (National Museum of Racing), on peut légitiment être étonné par la place prise par la "reconstitution» de l'ambiance des courses hippiques (chevaux grandeur nature sortant de l'autostart, affichage des paris), en fort contraste avec les salles consacrées aux œuvres d'art, qui reprennent tous les codes du monde muséal (fig. 9, fig. 10). Quant aux témoignages sociologiques (casaques, trophées, pèse-jockey, etc.) force est de constater, à Saratoga comme à Newmarket au Royaume-Uni (National Horseracing Museum ${ }^{16}$ ), qu'ils perdent largement leur puissance évocatrice de témoignage lorsqu'ils sont présentés sous vitrine, à la manière d'objets d'art à part entière. 
Figure 9

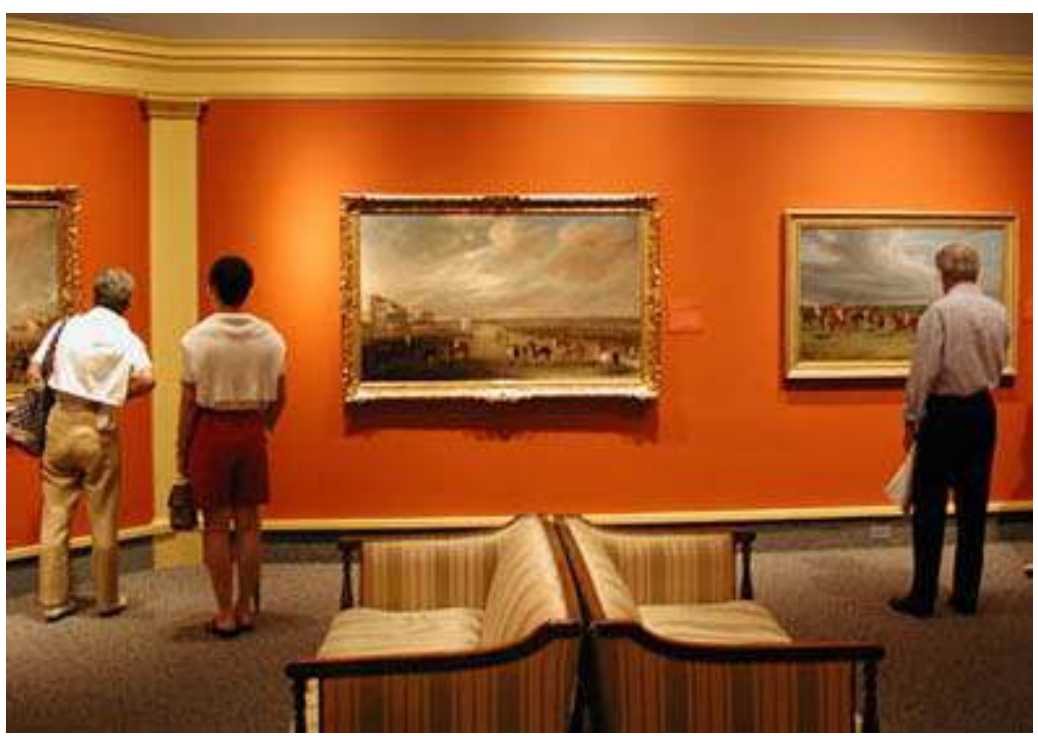

NATIONAL MUSEUM OF RACINg DE SARATOgA.

(C) NATIONAL MUSEUM OF RACINg.

Figure 10

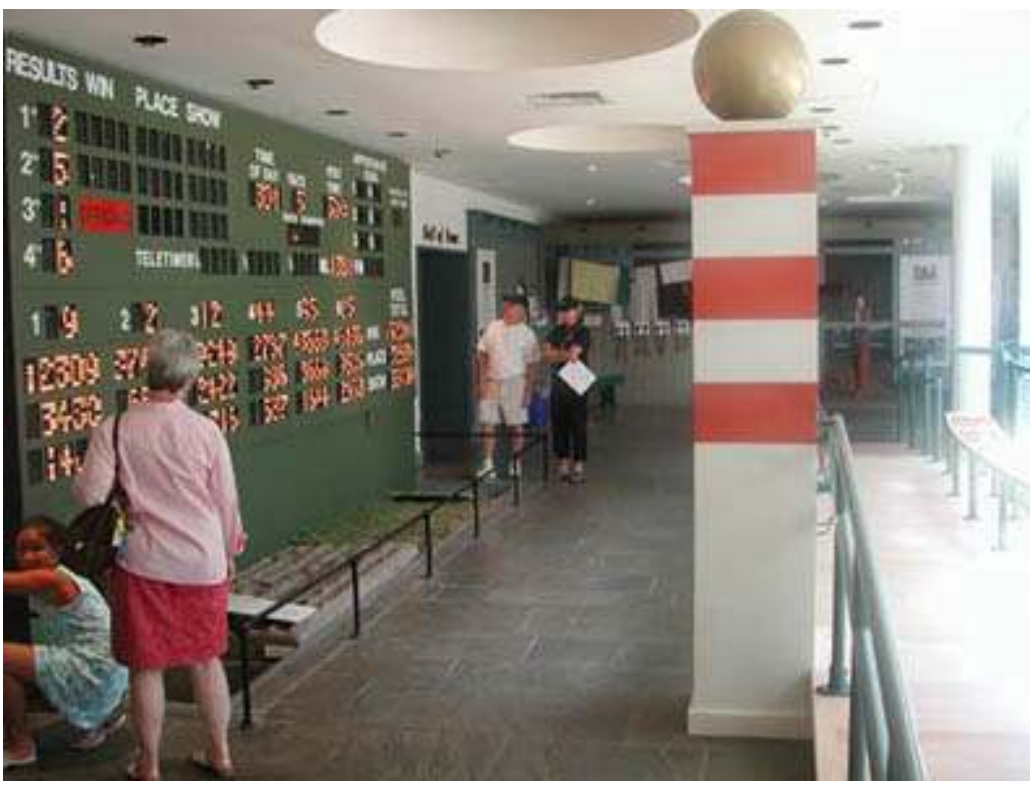

NATIONAL MUSEUM OF RACINg DE SARATOgA.

(C) National Museum of Racing.

De tous ces exemples, musées et monuments du Centre des monuments nationaux confondus, que retenir? Que la mémoire du cheval et de ses usages recouvre de facto l'œuvre d'art et le bien culturel, et que leur cohabitation nécessaire oblige à penser des modes d'exposition différents, si possible au sein d'un lieu évocateur dont la lecture ne sera pas perturbée par la présentation de collections. Il faut dans ce cas se résoudre à ne pas tout dire, ne pas vouloir dessiner l'évolution de la statue équestre à la ronde-bosse d'amateur de courses ou les progrès de l'anatomie équine jusqu'à la représentation plus 
exacte du galop des courses, même si bien évidemment, l'évolution de la représentation du cheval permet de comprendre la variété des témoignages sociologiques. Un axe de réflexion serait peut-être à creuser : celui de la mise en situation d'œuvres de qualité relativement secondaire au sein d'un intérieur d'amateur de courses, tel que de nombreuses aquarelles du début $d u \mathrm{XIX}^{\mathrm{e}}$ siècle l'évoquent ${ }^{17}$. Ainsi se trouverait peut-être effacée la complexité de la présentation de ces œuvres d'art qui n'ont jamais été conçues pour être admirées en tant qu'œuvres, mais dont la valeur demeurait essentiellement décorative et attestait surtout socialement du goût pour le cheval.

\section{NOTES}

1. - LOISEL, Germain. «Le château de Bouges », tiré à part de Monuments historiques, n¹93, 1994.

2. - COCHET, Vincent. Le château de Bouges. Paris : Éditions du patrimoine, «Itinéraires », 2004.

3. - LIÉVAUX, Pascal. Les écuries des châteaux français. Paris : Éditions du patrimoine, 2009.

4. - L'inventaire scientifique des harnachements a été complété et enrichi par Céline Do Paço, dans le cadre d'un stage en 2011.

5. - Préparation riche de protéines, à base d'avoine et d'orge.

6. - LIBOUREL, Jean-Louis. « Les voitures ». Dans Le château de Bouges, op. cit., p. 57-60.

7. - Ibid.

8. - LIÉVAUX, Pascal, op. cit..

9. - LOUIS, P. « Maisons au temps du Comte d'Artois. III : l'écurie anglaise : les chevaux de course de Monseigneur ». Dans Les cahiers de Maisons, n²0, 1991, p. 10-14.

10. - BARREAU, Jacques. Maisons-Laffitte : cité du cheval. Saint-Cyr-sur-Loire : A. Sutton, 2007, p. 11. 11. - Ibid.

12. - Le projet détaillé du contenu des salles et des objectifs du musée se trouve conservé à la médiathèque de l'Architecture et du Patrimoine (cote : 0081/078/0125).

13. - MUSÉE DU CHEVAL DE COURSES, Dossier de presse, 1990.

14. - MUSÉE DU CHEVAL DE COURSES, Dossier de presse, 1990.

15. - Notamment Charles Bargue ( ? -1883), d'après Alfred de Dreux (1810-1860), Portrait de l'étalon Atalanta ou Émile Lassalle (1813-1871), d'après Alfred de Dreux (1810-1860), Cheval emporté, 1860.

16. - Pour les figures 9 et 10, voir le site : http://www.racingmuseum.org/.

17. - DAVIDSON, Gail S., GERE, Charlotte, MCCARRON-CATES, Floramae et MARCHESSEAU, Daniel. Intérieurs romantiques : aquarelles 1820-1890. Cat. exp. Paris, musée de la Vie romantique, sept. 2012janv. 2013. Paris : Paris Musées, 2012, p. 186. STÖCKLER, E. Un Salon de sportsman, 1856 (CooperHewitt, National Design Museum, Smithsonian, New York). 


\section{RÉSUMÉS}

À travers les exemples des collections du château de Bouges (collections d'Henri Viguier, président du Cercle de l'Étrier) et du château de Maisons (collection constituée par l'Association pour le musée du cheval de courses), ce bref aperçu de chacune des deux collections présente leurs spécificités ainsi que leurs problématiques tantôt patrimoniales, tantôt muséales. Constituées selon des axes différents, leur valorisation pose de nouvelles questions : de quelle façon une écurie désormais vide de chevaux doit-elle être conservée ? Quel type de musée est-il possible d'envisager au sein des monuments?

The equestrian collections of the "Centre des Monuments Nationaux" at Bouges and Maisons. Looking at examples from the castle of Bouges (the collection of Henry Viguier, who was president of the Cercle de l'Étrier) and from the Maisons chateau (a collection put together by the association for a horse-racing museum), this brief overview of the collections presents their specificities and looks at some of the preservation and museum issues that the collections raise. Constituted in different ways, their interpretation poses new questions: how can a stable without horses be preserved? what type of museum is it possible to envisage inside a protected monument?

\section{INDEX}

Keywords : horse teams, harnessing, horse-drawn vehicle, stable, saddlery, horse-related collections

Mots-clés : attelage, harnachement, voiture hippomobile, écurie, sellerie, collections hippiques

\section{AUTEURS}

\section{MORWENA JOLY-PARVEX}

conservateur du patrimoine, chef du département de la conservation des collections, Direction scientifique, Centre des monuments nationaux morwena.joly-parvex@monuments-nationaux.fr

\section{GRÉGOIRE CUNY}

chargés d'études

\section{CÉLINE DO PAÇO}

historienne de l'art 